\title{
A Survey of Computer Network Management Technology
}

\author{
Tan Li \\ Chongqing Three Gorges University, Chongqing,China
}

Keywords: Computer; Network management; Technical; Network management technology

\begin{abstract}
With the development of computer technology and the internet, the administration and maintenance of computer network have acquired crucial importance, especially so in large computer network. Being a key technique in computer network, network management has an immediate impact on the efficiency of network operation. As a result, to ensure its stability and reliability is fundamental to an efficient network operation. This paper, from the perspective of the concept and development of computer management, takes an analysis of its current problems and proposes several solutions with wide application.
\end{abstract}

\section{The Concept of Computer Network Management Technology}

It refers to the centralized management of the resources on the internet through some network management programs, including the management of configuration, function, accounting, problem, operating process and variable data.

\section{The Present Technologies of the Computer Network Management}

Network management, as a science subject has been highlighted since 1980s. Many international organizations of standards, forums and research institutes have issued some kinds of standards, or agreement to conduct the network management and designs. Each one has its own properties, but no one has a universally accepted standards. Up to now, there are only three main network management technologies widely used. The first one is SNMP. Invented by Internet Company, SNMP is specially designed for internet management. It is easy to run and practical to use, so SNMP has become the practical standard for the internet world. However, due to its short time development, it also has some insufficiencies, which makes it hard be used in complex network management, only in the TCP / IP management. Furthermore, SNMP is not powerful enough to guarantee the security of the computer network. The second is CMIP. It can provide an all-round support for a complete network management, which is more advanced in technology and better completed in criteria. The best privilege is that the variable data in the contract is designed for the data on the terminals, but also for finishing some tasks of the network management. Anyhow, it is designed mainly to make up the insufficiencies of SNMP, and it is so complicated and expensive that it can't be accepted widely. The third is distributed object network management technology, which is generated from the applying process of CORBA on the internet. All the management application and elements are viewed as the distributed objects. And the interaction of the distributed objects is the task of the internet management. The distinguished properties are that it screened the differences of the program languages, internet contracts and operating systems. It is more incisive and easily see-through so that it can be researched and developed more easily and used more widely. SNMP and CMIP with their own properties appeal to different clients, so they can not be replaced 
by either of them for a long period. It is also impossible for the system of CORBA to replace them, because the time, cost and human resources for the research and development are so mass. Consequently, the research for the combination of the CORBA, SNMP and CMIP is suggested for the network management of the third technology.

\section{Application of the network management technology}

The aim of network management technology is to guarantee the internet and the network system to operate to some extent stably, reliably and efficiently, namely to make sure all the network resources are running smoothly and to satisfy the needs of the clients. In the past, there were some simple devices to help the network administrator to manage the resources. But now with the development of the internet, a powerful and easy-to-use network management technology is needed urgently to cope with the enormous and complicated internet resources. Only this kind of technology can fulfill the aim.

\subsection{Introduction of WBM technology}

Since the internet business is increasing, and the intranet technology is becoming popular, some major network companies attempt to use MIS technology in a new way, hence the Web-Based Management technology. As a complete new network management mode (based on Web network mode), its strong vitality, special flexibility and good serviceability are welcomed by a large number of technicians and clients, which was commended as "a final solution to the network management".

WBM technology based on the functions of the Web and its management technology, has greater capability than the traditional devices for the network administrators. WBM allows the administrator to use any kinds of Web browser, to allocate quickly at any point of the network, to control, store and withdraw any items on it. As a result, the administrators work is no longer confined to their offices; at the same time many operating problems caused by the different computer systems can also be resolved. Furthermore, WBM provides quicker and easier user interface for the internet log-in than the traditional driver. The browser and Web surface are familiar to the WWW clients, so the cost for the training of operation is much reduced and the network operation information is possibly well used by more and more internet clients. Therefore, we can say it is "a final solution to the network management".

3.2 Design of the network management system based on WBM technology

(1) Design Objective

The objective of the design is to develop Chinese network management system software in the "Web" pattern with independent copyright on the basis of campus network. By employing the advanced WBM technology and efficient algorithm, the software is expected to reach the level of the same products abroad.

This network management system will provide a complete set of solution based on WEB. Its effective resource management of distributed IP network enables the user to carry out strain type management and control to network, equipment as well as relevant system and service via WEB browser from anywhere. In this condition, the best operating status of the network resources, availability and reliability of the network can be guaranteed.

(2) System Architecture

The system is designed in the current technical conditions and with a view to technology development trend while taking advanced products abroad of the same kind as references. The three-tier architecture used by us, which has incorporated advanced WBM technology, offers the administrator a simple and convenient way of management. 


\section{(3) the Characteristics of the Three-tier Architecture}

The software responsible for management task works in background process way as middle tier to carry out polling of network equipment and collection of fault information.

The management middleware resides in between network equipment and browser, thus users just need to manage the homepage of middle tier in order to access the equipment.

The management middleware relay forwards management information and makes protocol conversion between SNMP and HTTP. No change is necessary for the equipment in three-tier architecture.

\subsection{The Design of Network Topology Discovery Algorithm}

To effectively manage the network, the administrator needs an intuitive and friendly user interface, of which the fundamental role is unfold the topological relation of network equipment graphically before the users' eyes, namely, topology discovery. At present, the topology discovery algorithm in widespread adoption is based on SNMP. It is very effective and fast to some extent, but there exists a defect [3]. That is, in a particular field, all information on subnetwork depend on that the equipment posses the characteristic of SNMP. Therefore, the algorithm will become invalid in a system incompatible with SNMP. Moreover, the SNMP function may be closed due to lack of attention to network management or for security reasons, as a result, imperfection of topology will be caused by the difficulty in obtaining MIB values of equipment. With the purpose of resolving this problem, an improvement of the algorithm above will be discussed in the following--- topology discovery based on ICMP.

\subsection{PING and the Establishment of Routing}

PING is mainly used to send messages, and to wait for answers. PING got its name because it's a simple echoing agreement, using ICMP request and response to send messages. PING is mainly used by the system programmers to diagnose and debug, and it can be realized through the following processes. First, send an ICMP message to the target machine, and then wait for the response from it, until overtime. If the response messages can be received, it proves that the target machine works well. Then the program withdraws.

The function of Routing is to use TTL domain in IP. At first, the source defines the TTL value of IP as zero, and sends messages to the informationsink. After finding out that the TTL value is zero, the first gateway rejects the messages and sends an overtime-typed ICMP message to the source, which then analyzes the message. Therefore the first gateway address is created. Then the source sends the message with TTL 1 to the informationsink. The first gateway reduces its TTL value to zero, and then sends it to the second gateway, which rejects the message, and sends the overtime-typed ICMP message to the source, after finding out that the TTL value is zero. Therefore the second gateway address is created. When the message reaches the informationsink, the Routing to the informationsink is created.

\subsection{Processes to Realize Network Topology Discovery Algorithm}

1) In the confined IP space, take turns to detect each IP address, by using PING, and record the detected IP addresses in the list of IP addresses.

2) To trace route every IP address, to record the routing of these IP addresses, and to add the gateway addresses into the IP list.

3) For every IP address in the list, find out its corresponding subnet mask.

4) According to the submask, find out the corresponding subnet website, and its network, adding each subnet to the list.

5) Try to get the corresponding Domain Name of each IP address. The same Domain Name means that the same network facility has many IP addresses, i.e. many network interfaces. 
6) Create the connection profile according to the routing got in the $2^{\text {nd }}$ step and the subnet got in the $4^{\text {th }}$ step.

\section{Conclusion}

At present, the application of computer network is in an explosive growth period. With the rapidly expanding network, the network complexity is also aggravating. With the development of network technology and the network business application, it becomes more and more important to manage and maintain the computer network. We must pay more attention and strive to manage the network well, to let the highly efficient and safe network promote the economic and social development.

\section{Reference}

[1]Tan Xianhai. 2006. Network Program Technology and Application [M]. Beijing: Tsinghua University Press.

[2]Tian Yuan. 2009. Security on Internet [M]. Beijing: People's Telecommunication Press.

[1]Sun Jianhua. 2005. Interconnecting Network Technology [M]. Superstar Library.

[3]Ye Shuhua. 2006. A Course Book for Network Program Technology [M]. Beijing: People's Telecommunication Press.

[4]Chen Xingyuan. 2008. An Agreement for Security of Internet Communication [M].Beijing: Higher Education Press.

[5]Qin Zheng. 2007. Network Application Psychology [M]. Beijing: Science Press.

[6]Li Fuliang. 2007. An Intensive Study of Network Application [M]. Beijing: Electronics Industry Press.

[7]Zhang Baosheng. 2007. Innovations and Competitiveness of Network Technology [M]. Beijing: Business Management Press.

[8]Zhang Pusheng. 2010. Computer Network Technology [M]. Beijing: Science Press.

[9]Niu Yan. 2010. Computer Network Technology and the Application [M]. Beijing: Tsinghua University Press. 